\title{
Narrow BP Active Filters with Reduced Noise and Sensitivity Using Current Conveyors
}

\author{
Bojan Gašpar and Dražen Jurišić \\ Faculty of Electrical Engineering and Computing \\ University of Zagreb \\ Unska 3, HR-10000 Zagreb, Croatia \\ e-mail: \{bojan.gaspar|drazen.jurisic\}@fer.hr
}

\author{
Budimir Lutovac \\ Faculty of Electrical Engineering \\ University of Montenegro \\ George Washington bb, ME-81000 Podgorica, Montenegro \\ e-mail: budo@ac.me
}

\begin{abstract}
This paper presents the low-sensitivity realization of narrow band-pass analog active filters using second-generation current conveyors. It is known that filters using multiplefeedback structures have reduced sensitivities to passive component tolerances. It was shown that using the follow-the leader (FLF) topology, the sensitivity of current transfer function magnitude was reduced when compared to the common cascade topology (CAS). The sensitivity reduction happens in the current mode in the same way as in the voltage-mode. Besides sensitivity, the output thermal noise is also reduced using the FLF topology. As an example, the design procedure and the Monte Carlo sensitivity, as well as output thermal noise analysis using PSpice were performed for a 4th-order Butterworth narrow BP filter.
\end{abstract}

Keywords-current mode filters; multiple-feedback structures, low sensitivity; low noise; second-generation current conveyor

\section{INTRODUCTION}

The realization of narrow band-pass (BP) filters yields very high pole Q-factor values, resulting from the application of the low-pass-to-band-pass (LP-BP) transformation to an all-pole low-pass (LP) function. High pole Q-factors produce high filter-magnitude sensitivity to the variation of passive components. In this paper, we compare a cascade (CAS) structure [Fig. 1(a)], to the filters using a follow-the leaderfeedback (FLF) structure [Fig. 1(b)] in order to reduce high passive component sensitivity (see [1][2]). The negative feedback of signals shown in Fig. 1(b) is universal and can be applied in both voltage and current modes. Besides, lowering sensitivity it is also applicable for lowering output thermal noise.

Current-mode approach offers high-frequency application because of no limitation on the GBW product when compared to traditional voltage-mode approach [3]. Besides, it does not (a)
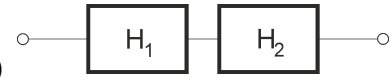

$\beta_{2}$

(b)

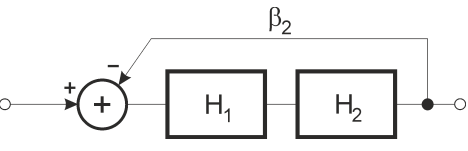

Figure 1. (a) CAS structure. (b) FLF structure. need high-voltage gains and provides circuits with good performance in terms of speed, bandwidth and accuracy. Inside the current-mode circuits, the second-generation current conveyor (CCII) can be considered the basic active circuit building block [3].

\section{THE HIGH-ORDER NARROW BP FILTER REALIZATION}

Each high-order (i.e. $n^{\text {th }}$-order; $n>2$ ) symmetrical BP transfer function can be written as a product of fourth-order factors if $n=4 k ; k=1,2, \ldots$ and eventually one second-order factor if $n=2(2 k+1) ; k=1,2, \ldots$.

A. Second-order BP Filters using LP-BP Transformation

If we apply the LP-BP transformation

$$
S \rightarrow \frac{s^{2}+\omega_{0}^{2}}{B s}
$$

to the first-order LP prototype

$$
H_{L P}(S)=k_{L P} \cdot \frac{\gamma}{S+\gamma}
$$

we obtain the second-order BP transfer function

$$
H_{B P}(s)=\frac{k_{L P} \cdot \gamma B \cdot s}{s^{2}+\gamma B \cdot s+\omega_{0}^{2}}=\frac{k_{B P} \cdot\left(\omega_{p} / q_{p}\right) \cdot s}{s^{2}+\left(\omega_{p} / q_{p}\right) \cdot s+\omega_{p}^{2}} .
$$

From (3) second-order BP pole parameters readily follow:

$$
\omega_{p}=\omega_{0} ; q_{p}=\omega_{0} /(\gamma B) ; k_{B P}=k_{L P} .
$$

Note that $\omega_{0}$ is a center frequency and $B$ is a bandwidth of a BP filter. [It is convenient to start with $\gamma=1$ in (2)].

\section{B. Fourth-order BP Filters using LP-BP Transformation}

Cascade Structure (CAS). In this section we investigate the fourth-order factors, and we propose the building block that covers most of the BP filter topology. If we apply the LP-BP transformation (1) to the second-order LP prototype: 


$$
H_{L P}(S)=\frac{k_{L P} \cdot \omega_{L P}^{2}}{S^{2}+\left(\omega_{L P} / q_{L P}\right) S+\omega_{L P}^{2}}
$$

we obtain the fourth-order BP transfer function which can be represented as product of two second-order factors that have a form:

$$
F_{4}(s)=H_{1}(s) \cdot H_{2}(s)=\prod_{i=1}^{2} \frac{k_{i} \cdot\left(\omega_{0 i} / q_{i}\right) \cdot s}{s^{2}+\left(\omega_{0 i} / q_{i}\right) \cdot s+\omega_{0 i}^{2}} .
$$

Equation (6) represents the cascade realization of a fourthorder BP filter. The product (6) contains the factor pairs having equal Q-factors $q_{1}=q_{2}=q$ defined by

$$
q=\sqrt{\frac{\left(4+B^{2} \omega_{L P}^{2} / \omega_{0}^{2}\right)+\sqrt{\left(4+B^{2} \omega_{L P}^{2} / \omega_{0}^{2}\right)^{2}-4\left(B^{2} \omega_{L P}^{2}\right) /\left(q_{L P}^{2} \omega_{0}^{2}\right)}}{2\left(B^{2} \omega_{L P}^{2}\right) /\left(q_{L P}^{2} \omega_{0}^{2}\right)}}
$$

and pole frequency pairs $\omega_{01}, \omega_{02}$, which are given by

$$
\frac{\omega_{01}}{\omega_{0}}=\frac{\omega_{0}}{\omega_{02}}=\frac{B q \omega_{L P} / \omega_{0}+\sqrt{\left(B q \omega_{L P} / \omega_{0}\right)^{2}-4 q_{L P}^{2}}}{2 q_{L P}} .
$$

Note that $\omega_{01} \omega_{02}=\omega_{0}$, where $\omega_{0}$ is the center frequency of a BP filter. Equations (7) and (8) are well known and referred to as "Geffe equations". They provide all parameters needed to construct fourth-order BP filter in cascade form (see Fig. 1(a)).

Follow the Leader Feedback Structure (FLF). It is well known that the overall sensitivity of a BP filter is minimal if each fourth-order factor defined by (6) is realized by a socalled "Biquartic structure" (having also the name FLF) as shown in Fig. 1(b) with identical second-order blocks [1]. The transfer function of a Biquartic BP structure is:

$$
F_{4}(s)=\frac{H_{1 B}(s) H_{2 B}(s)}{1+\beta_{2} H_{1 B}(s) H_{2 B}(s)} ; H_{i B}(s)=\frac{k_{i B}\left(\omega_{0} / q_{B}\right) s}{s^{2}+\left(\omega_{0} / q_{B}\right) s+\omega_{0}^{2}},(9)
$$

where $H_{i B}(s)(i=1,2)$ represent the transfer function of each second-order BP sub-network, and $\beta_{2}$ represents a negative feedback factor ${ }^{1}$. The design parameters needed for realizing the fourth-order biquartic structure from a given fourth-order cascade structure [i.e. to construct fourth-order BP filter in FLF form as in Fig. 1(b)] follow from equating (6) and (9). The design parameters are:

$$
\begin{aligned}
& q_{B}=2 q \omega_{0} /\left(\omega_{01}+\omega_{02}\right) ; k_{1 B} k_{2 B}=k_{1} k_{2} q_{B}^{2} / q^{2} ; \\
& \beta_{2} k_{1 B} k_{2 B}=\left(4 q^{2}-1\right) \cdot\left(\omega_{01}-\omega_{02}\right)^{2} /\left(\omega_{01}+\omega_{02}\right)^{2} .
\end{aligned}
$$

\section{Example}

As an illustration the design procedure of the two versions of the fourth-order BP filters shown in Fig. 1 is considered. The filter is derived from the second-order Butterworth LP filter prototype with normalized $3 \mathrm{~dB}$ cut-off frequency $\omega_{c}=1$, having normalized pole frequency $\omega_{p}=1[\mathrm{rad} / \mathrm{s}]$, and the pole Qfactor $q_{\mathrm{p}}=1 / \sqrt{ } 2$. The BP center frequency is $\omega_{0}=2 \pi \cdot 10^{4}[\mathrm{rad} / \mathrm{s}]$,

\footnotetext{
${ }^{1} \beta_{2}$ has index 2 because it takes signal after second block.
}

and the bandwidth $B=2 \pi \cdot 500[\mathrm{rad} / \mathrm{s}]$, or normalized, $B_{n}=B / \omega_{0}=$ 0.05 (selective BP filter). For cascade structure, using (7) and (8) we calculate: $q=28.2887 ; \omega_{01}=63952.6[\mathrm{rad} / \mathrm{s}] ; \omega_{02}=61730.8$ [rad/s]; and we choose $k_{1}=k_{2}=0.5$ (we choose $k_{i}<1, i=1,2$ to obtain realizable Biquad in Section III). For FLF structure using (10) we calculate: $q_{B}=28.28427 ; \beta_{2}=4.00125$; and $k_{1 \mathrm{~B}}=k_{2 \mathrm{~B}}=0.5$. Note that for the narrow BP filters, from (8) we have obtained high pole $\mathrm{Q}$ factors $\left(q\right.$ and $\left.q_{B}\right)$ as a consequence of LP-BP transformation. For narrower pass band even larger pole Qs (of the order of hundreds) are obtained.

\section{BP FILTER REALIZATIONS}

Consider current-mode BP biquadratic filter section shown in Fig. 2 [4] using two second generation current conveyors and only two grounded capacitors (i.e. canonic number of capacitors). Current transfer function for the filter section Fig. 2 is defined by:

$$
H_{i}(s)=\frac{I_{o u t}(s)}{I_{\text {in }}(s)}=-\frac{k_{B P}\left(\omega_{p} / q_{p}\right) s}{s^{2}+\left(\omega_{p} / q_{p}\right) s+\omega_{p}^{2}},
$$

where parameters as function of components are defined by:

$$
\omega_{p}=\frac{1}{\sqrt{C_{1} R_{2} R_{3} C_{4}}} ; q_{p}=\frac{\sqrt{C_{1} R_{2} R_{3} C_{4}}}{\left(R_{2}+R_{3}\right) C_{4}} ; k_{B P}=\frac{R_{3}}{R_{2}+R_{3}} .
$$

The step-by-step design procedure for the circuit in Fig. 2 follows:

i) Choose $C_{4}$ and calculate: $R_{3}=k_{B P} /\left(C_{4} \omega_{p} q_{p}\right)$;

ii) From $k_{B P}$ calculate: $R_{2}=R_{3}\left(1 / k_{B P}-1\right)$;

iii) Calculate: $C_{1}=1 /\left(\omega_{p}^{2} R_{2} R_{3} C_{4}\right)$.

Using design equations (13)-(15) and pole parameters from above example we obtain the following component values given in Table I for both CAS and FLF filter circuits presented in Fig. 4 and Fig. 5, respectively.

Second-generation current conveyor with designated port voltages and currents is shown in Fig. 3(a), and is defined by (16). Its equivalent schematic which we use in PSpice for idealized simulation is shown in Fig. 3(b) with unity gain current controlled current source (CCCS); in PSpice component " $\mathrm{E}$ " is used. It is connected to voltage controlled voltage source (VCVS); in PSpice component " $F$ " is used. For the positive conveyor CCII+ there is +1 in matrix (16), i.e. $I_{z}=I_{x}$ with references as in Fig. 3(a), whereas for the CCII- we have -1 . In our analysis we have used only positive $\mathrm{CCII}+$, they are also convenient to be realized in discrete form using AD844.

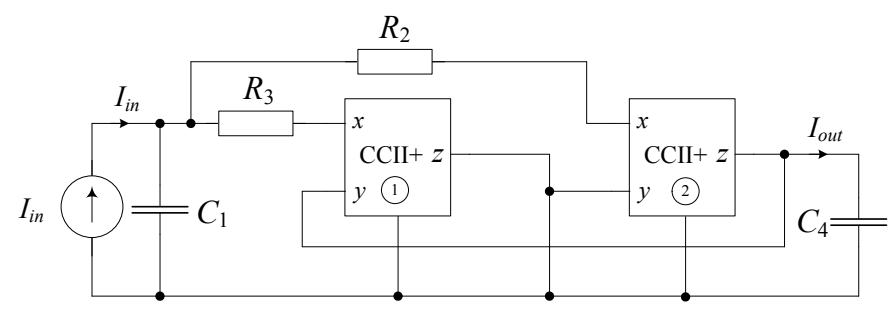

Figure 2. Current-mode BP biquadratic section using two CCII+. 
TABLE I. ELEMENT VALUES OF CAS (IN Fig. 4), AND FLF (IN Fig. 5) FILTERS (CAPACITORS IN nF, RESISTORS IN $\Omega$ ).

\begin{tabular}{|c|c|c|c|c|c|c|}
\hline Type & \multicolumn{7}{|c|}{ Element values } \\
\hline \multirow{4}{*}{ CAS } & $C_{1}$ & $R_{2}$ & $R_{3}$ & $C_{4}$ & $R_{8}$ & $R_{9}$ \\
\cline { 2 - 7 } & 3200 & 276.375 & 276.375 & 1 & 8000 & 1000 \\
\cline { 2 - 7 } & $C_{5}$ & $R_{6}$ & $R_{7}$ & $C_{8}$ & & \\
\cline { 2 - 7 } & 3200 & 286.322 & 286.322 & 1 & & \\
\hline \multirow{2}{*}{ FLF } & $C_{1}, C_{5}$ & $R_{2}, R_{6}$ & $R_{3}, R_{7}$ & $C_{4}, C_{8}$ & $R_{8}, R_{10}$ & $R_{9}, R_{11}$ \\
\cline { 2 - 7 } & 3200 & 281.305 & 281.305 & 1 & 8000 & 1000 \\
\hline
\end{tabular}

(a)

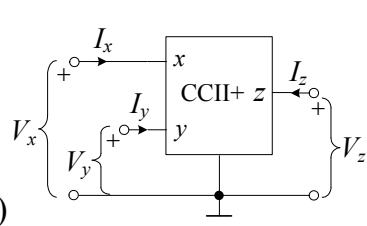

(b)

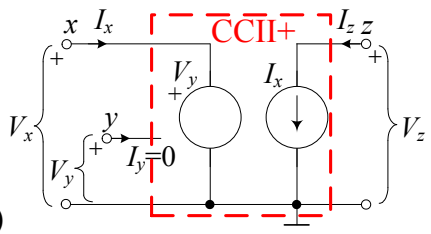

Figure 3. (a) CCII+ with designated port voltages and currents. (b) Model of ideal CCII+ in PSpice.

$$
\left[\begin{array}{l}
I_{y} \\
V_{x} \\
I_{z}
\end{array}\right]=\left[\begin{array}{ccc}
0 & 0 & 0 \\
1 & 0 & 0 \\
0 & \pm 1 & 0
\end{array}\right]\left[\begin{array}{l}
V_{y} \\
I_{x} \\
V_{z}
\end{array}\right]
$$

In the circuit in Fig. 4, CCII+ no. 3 and 5 function as "current followers" (and current inverters) to convey the current through $C_{4}$ to the output current source. The CCII+ no. 7 with resistors $R_{8}$ and $R_{9}$ form the current amplifier. In the circuit in Fig. 5, CCII+ no. 6 and 7 function as non-inverting current followers (and can be replaced by one CCII-). This is because the proper polarity of the current signal is needed to form negative feedback. Note that the addition of the signals in current-mode is simple; two currents: from the $z$ output of CCII+ no. $8\left(I_{\text {out }}\right)$ and input source $\left(I_{\text {in }}\right)$ are simply connected at one node and summed by the current Kirchhoff law (KCL).

\section{A. Sensitivity and Noise Analysis}

In what follows, we examine the sensitivity to tolerances of passive component values of the two current-mode filters discussed above realizing fourth-order BP filter whose amplitude characteristics $\alpha_{i}(\omega)=20 \log \left|H_{i}(j \omega)\right|[\mathrm{dB}]$, where $H_{i}(s)$ is the current transfer function, are shown in Fig. 6(a). Using the OrCAD PSpice 16 program [6] with Monte Carlo runs, we assume a zero-mean uniform distribution, and a 5\% standard deviation for all components. The obtained spread of responses for each filter is an indication of that filter's sensitivity to component tolerances.

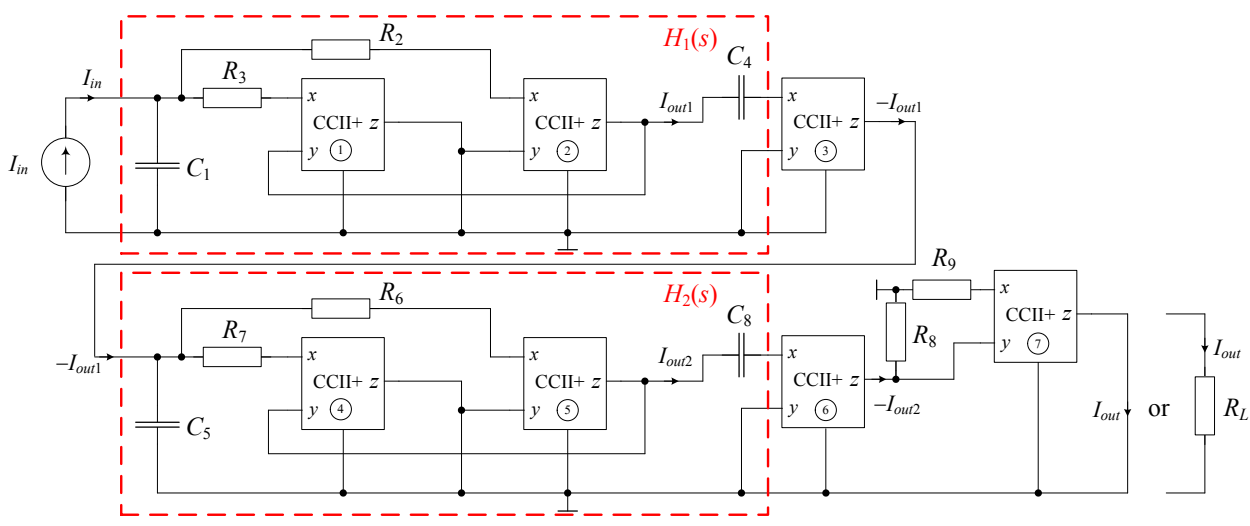

Figure 4. Current-mode fourth-order BP filter using cascade structure (CAS). $\left(R_{L}\right.$ is used when the output noise is measured.)

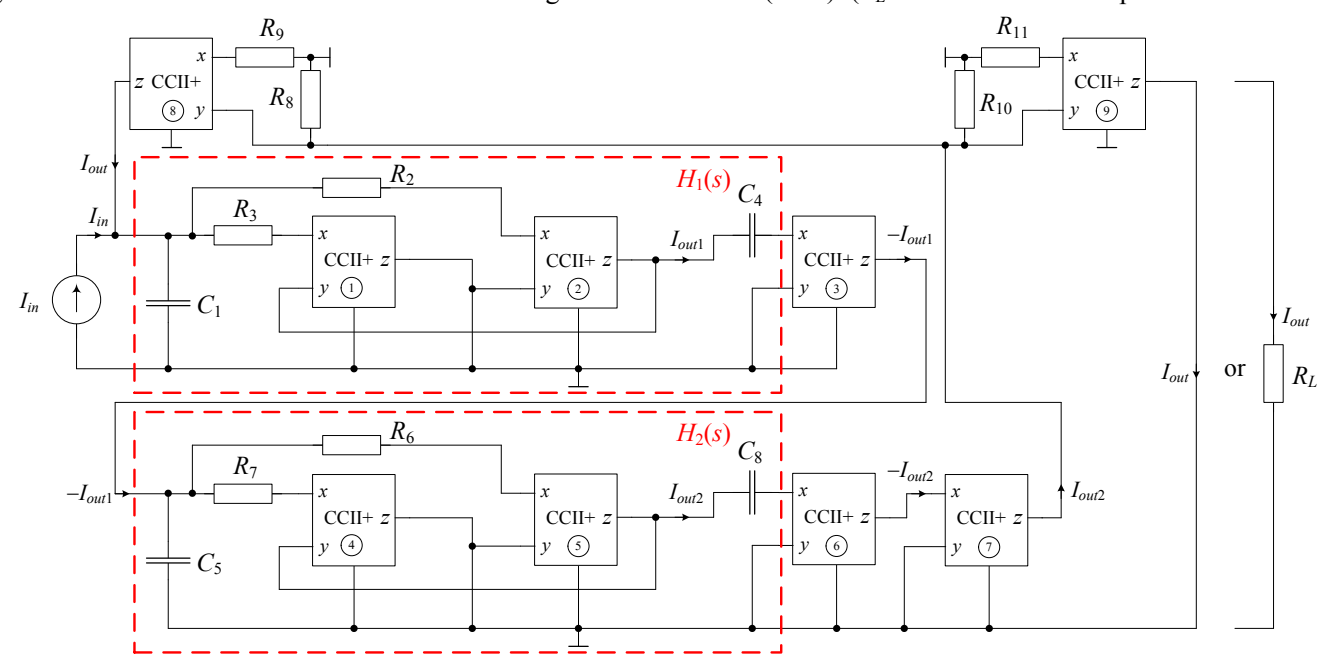

Figure 5. Current-mode fourth-order BP filter using follow-the-leader-feedback structure (FLF). ( $R_{L}$ is used when the output noise is measured.) 


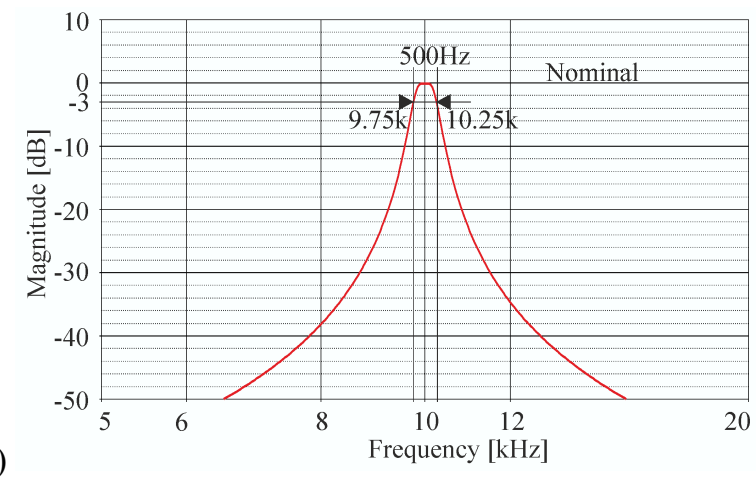

(a)

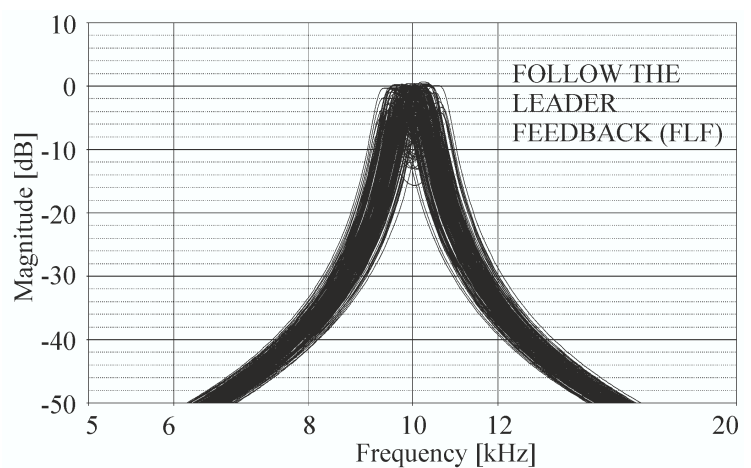

(b)
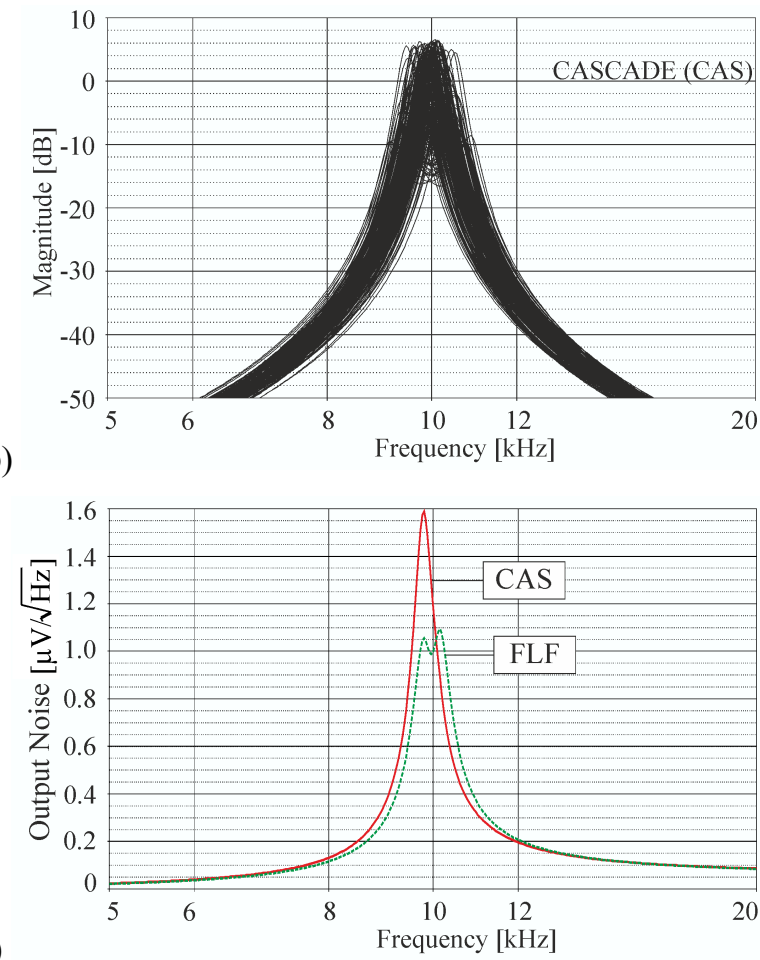

Figure 6. (a) Amplitude characteristics of the current transfer function $H_{i}(s)$ of fourth-order BP filter example. Monte Carlo runs of various realizations: (b) Cascaded filter (CAS) (Fig. 4). (c) Follow-the-leader-feedback filter (FLF) (Fig. 5). (d) Output noise spectral density as voltage on $R_{L}=50 \Omega$ terminating resistance.

The sensitivity of the CAS filter (see Fig. 4) is shown in Fig. 6(b), and that of FLF (see Fig. 5) in Fig. 6(c). As expected, lower sensitivity possesses the filter with negative feedback (FLF) although it has higher number of components. This is the same result as in the voltage-mode domain regarding sensitivity comparison of CAS and FLF structures.

To analyze output noise, both filters were simulated using the AD844/AD transimpedance opamps from Analog Devices. For this amplifier, its typical values given in the data book [7] are: $R_{x}=50 \Omega, R_{z}=3 \mathrm{M} \Omega$ and $C_{z}=4.5 \mathrm{pF}$. These values are also used in PSpice model of AD844/AD. Therefore to compensate for the input resistance $R_{x}$, we have reduced the values of resistors $R_{2}$ and $R_{3}$ (in Table I) for $50 \Omega$. At the output we have connected load resistor $R_{L}=50 \Omega$ (instead short circuit) which leads output current $I_{\text {out }}$ (see Figs. 4 and 5). The same situation would occur if we have connected the current follower at the output using $x$ node of AD844/AD. At the $R_{L}$ we then measure the output thermal noise spectral density as voltage, which is presented in Fig. 6(d). It is shown in Fig. 6(d) that the CAS circuit (Fig. 4) has higher noise than the FLF circuit (Fig. 5), although the former has lower number of active and passive components.

\section{CONCLUSIONS}

In this paper we compare two realizations of current-mode BP filter of fourth-order, which is common filter block in high-order BP filters. One is classical cascade realization; the second, referred to as FLF, uses negative feedback and two identical current-mode Biquads. It is demonstrated that negative feedback in FLF reduces the sensitivity to component tolerances of the circuits. The output noise of the two circuits is compared, and it is shown that lower noise has FLF filter, too. The improvement of sensitivity and noise features in both current- and voltage-mode approaches comes at price; FLF filter has more components then commonly used CAS filter.

\section{ACKNOWLEDGMENT}

This work has been financially supported by the bilateral project "Integrated Systems for Analog and Mixed Signal Processing-Active-RC filters for ADSL Communication Systems" between the Ministry of Science, Education and Sports of the Republic of Croatia and the Ministry of Science of the Republic of Montenegro.

\section{REFERENCES}

[1] N. Mijat and G. S. Moschytz, "Sensitivity of narrowband biquartic BP active filter block", In Proc. $5^{\text {th }}$ ISYNT, Sarajevo, 1984, pp.158-163.

[2] J. Tow, "Some results on generalized follow-the-leader-feedback active filters", IEEE Trans. on Circuits and Systems, vol. 25, p.p. 462-465, April, 1978.

[3] C. Toumazou, F. J. Lidgey and D. G. Haig, Analog IC Design: The Current Mode Approach, London, U.K.: Peter Peregrinus, 1990.

[4] G. Ferri and N. C. Guerrini, Low Voltage, Low Power CMOS Current Conveyors, Boston, U.S.: Kluwer Academic Publishers, 2003.

[5] R. Schaumann, M. S. Ghausi and K. R. Laker, Design of Analog Filters, Passive, Active RC, and Switched Capacitor, New Jersey: Prentice Hall, 1990.

[6] PSPICE Users Guide ver. 16.5, Cadence Design Systems, Inc. (Cadence), San Jose CA-USA, May 2011.

[7] ANALOG DEVICES: 'Linear Products Data Book' (Norwood, MA 02062, USA, 1990. 